\title{
When to Consider Immune Checkpoint Inhibitors in Oncogene-Driven Non-Small Cell Lung Cancer?
}

Laurent Mhanna, $M D^{1}$

Nicolas Guibert, MD, $P h D^{1}$

Julie Milia, $P h D^{1}$

Julien Mazieres, MD, $P h D^{1,2, *}$

\author{
Address \\ ${ }^{1}$ Pulmonology Department, Toulouse University Hospital, Université Paul Saba- \\ tier, Toulouse, France \\ ${ }^{*}, 2$ Thoracic Oncology Department, Hopital LARREY, CHU Toulouse, Chemin de \\ Pouvourville, 31059, Toulouse, France \\ Email: mazieres.j@chu-toulouse.fr
}

Published online: 6 June 2019

(C) The Author(s) 2019

This article is part of the Topical Collection on Lung Cancer

Keywords Immunotherapy $\cdot$ Oncogenic addiction $\cdot$ Targeted therapy $\cdot$ EGFR $\cdot$ ALK $\cdot$ Non-small cell lung cancer

\section{Opinion statement}

Targeted therapies and more recently immune checkpoint inhibitors (ICI) have transformed the treatment landscape of advanced NSCLC. Clinical trials investigating immune checkpoint inhibitors (ICI) have usually excluded patients with oncogenic drivers, so that the outcome of these agents in this population is poorly known. In patients with oncogenic addiction, targeted therapy remains clearly the best option, and the place of immunotherapy in this population has not been clearly defined yet.

Based on available data, we suggest that (i) immunotherapy single agent should be proposed only after exhaustion of more validated treatments, (ii) combinations of immunotherapy with targeted therapies are of interest provided that we can manage toxicity and find the best sequence, (iii) a combination of immunotherapy with chemotherapy may be appealing in patients pretreated with targeted agents. The best way to opt in for the best strategy will depend upon the identification of adequate biomarkers. New basic and clinical research is awaited in this field. 


\section{Introduction}

Lung cancer is the main cause of cancer-related mortality worldwide. Its management underwent significant transformation over the past 10-15 years leading to two new therapeutic strategies: targeted therapies and immunotherapy. Genotype-directed treatments targeting oncogenic addictions (EGFR, BRAF, and HER2 mutations, or ALK and ROS1 rearrangements) demonstrated high response rates and prolonged PFS.

Immunotherapy has been also recently developed in non-small cell lung cancer (NSCLC). The positive results of clinical trials assessing PD-1 and PD-L1 inhibitors in both metastatic and locally advanced stages revolutionized the treatment landscape of NSCLC [1-3]. In all the studies considering these agents however, only a minority of patients (15-20\%) derived a durable benefit and a new pattern of hyperprogressive patients has been identified, suggesting an urgent need for new biomarkers of both response and resistance.
The place taken by ICI in patients with oncogenic driver is actually debated, since they have been usually excluded from phase 3 immunotherapy trials. However, despite a usual dramatic and durable activity of targeted therapies, resistance inexorably develops [1]. Treatment options upon exhaustion of targeted therapies are limited, underscoring the need to explore the potential interest of ICI in these populations.

Another challenge is to further understand the biological drivers of inflammation and immune escape in NSCLC with oncogenic addiction and to identify subgroups deriving benefits.

We aim herein to review the main data available regarding the outcome of patients harboring an oncogenic addiction and treated with ICI either alone, in combination with chemotherapy or concomitantly to targeted therapy.

\section{What is the immunogenicity of lung cancer with oncogenic addiction?}

The evolution of tumors bearing a molecular alteration usually depends on a single dominant mechanism following the principle of oncogenic addiction, which has been described as the dependence of tumor cells upon the specific activity of an activated oncogene [4]. A single mutation or translocation is supposed to confer a survival advantage to the respective cells and it is usually isolated, explaining the low tumor mutation burden observed in these tumors [5], leading to less inflamed tumor microenvironments with death of tumor-

\section{Global population}

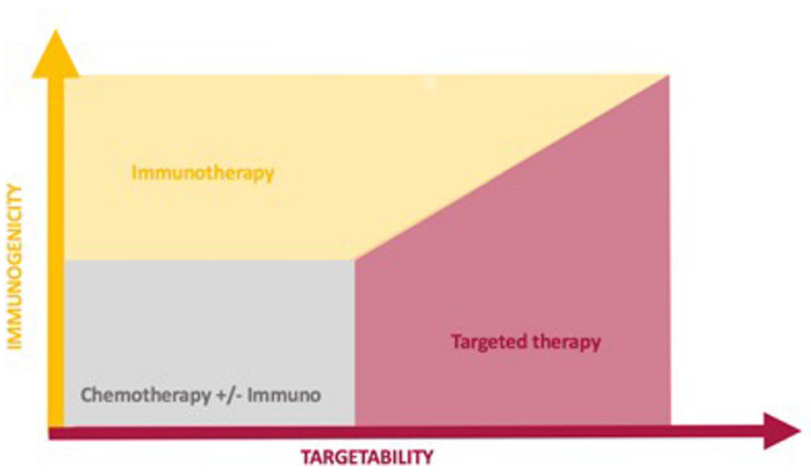

\section{Population with oncogenic addiction}

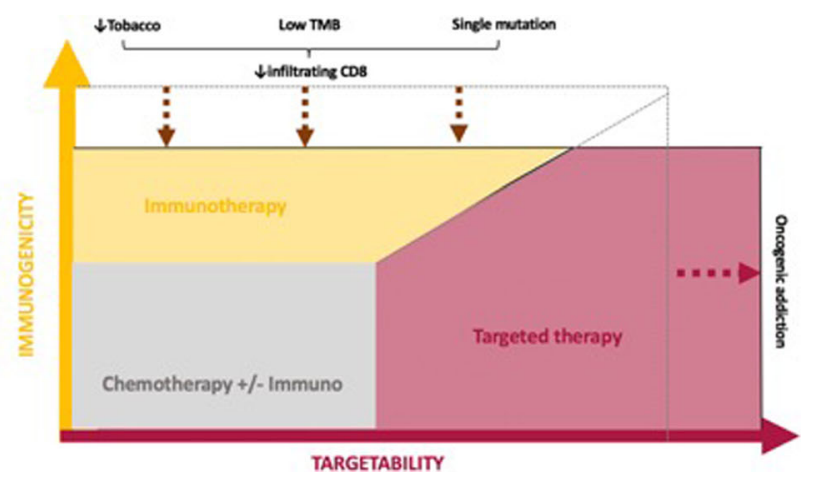

Fig. 1. Immunogenicity and sensitivity to targeted agents in situation of oncogenic addiction. 
infiltrating $\mathrm{CD}^{+}$lymphocytes, explaining the low response rate to PD-1 inhib-

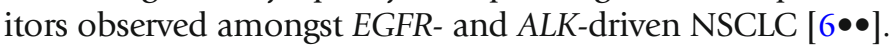

EGFR, BRAF ${ }^{\nu 600 E}$ mutations, ALK, ROS, or RET rearrangements but also $M E T$ exon 14 mutations are usually found in non-smokers, in contrast with KRAS and BRAF non V600E which are more likely found in smokers [7]. It is clearly established that tobacco exposure is associated with higher tumor mutation burden (TMB) and correlates with higher responsiveness to ICI, while non-smokers will less likely respond to these agents due to excluded, non-

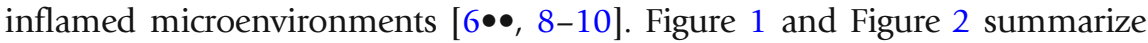
the sensitivity to ICI and targeted therapy regarding each oncogenic addiction.

\section{Anti-tumor activity of ICI as monotherapy according to each type of mutation}

\section{KRAS}

KRAS mutations are the most frequent molecular alterations encountered in advanced NSCLC and are correlated with both poor prognosis and poor outcomes under chemotherapy [11-13]. Moreover, all studies involving therapies targeting KRAS failed to demonstrate any clinical benefit up to now [14, 15] due notably to the complexity of its downstream signaling pathways.

ICI however clearly represent an attractive alternative in KRAS-mutated patients, since correlations between KRAS mutations and sustained responses to checkpoint inhibitors have systematically been reported in trials $[1,8]$. In a recent work, Miao et al. reported that clonal driver alterations in KRAS were associated with complete or partial response to ICI [16]. This relationship is likely due to a strong epidemiologic association with tobacco that generates a high mutation burden $[17,18]$.

In our retrospective IMMUNOTARGET cohort, we reported an interesting 3.2 months of median PFS in the overall KRAS population but failed to detect a

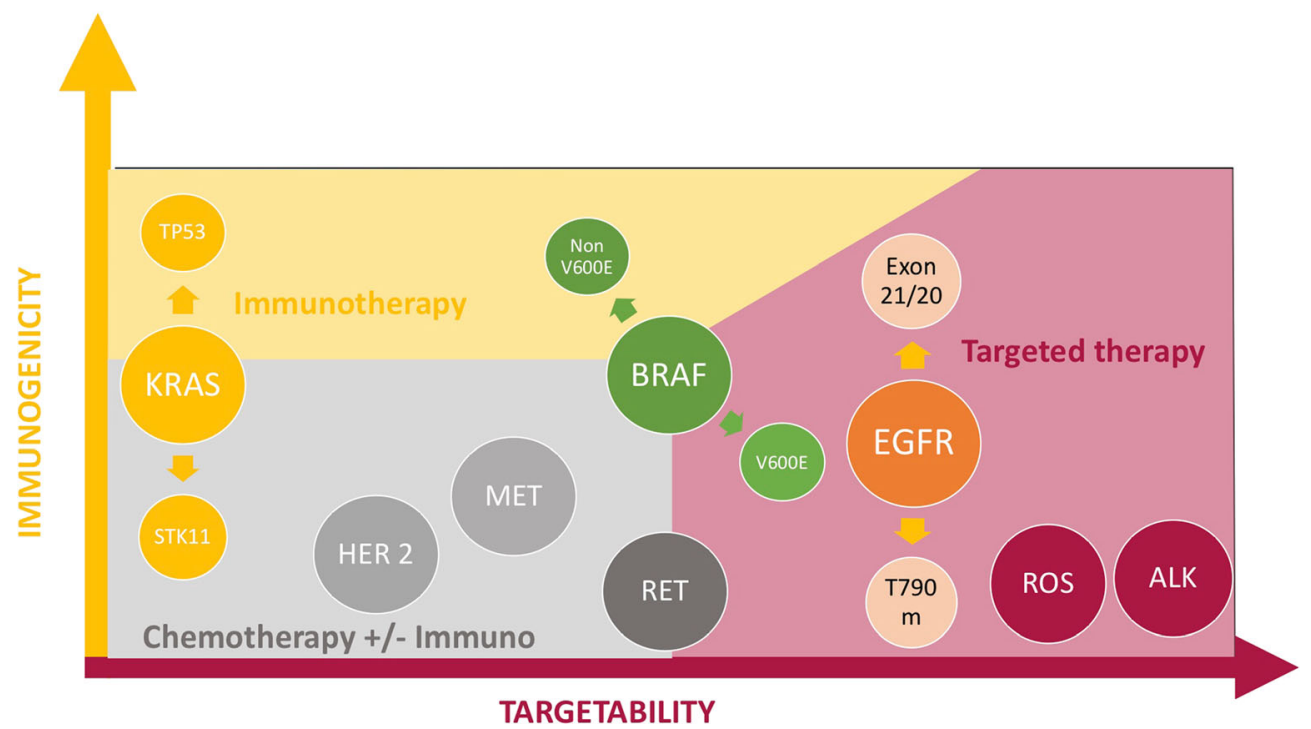

Fig. 2. Likelihood of sensitivity to ICI and/or genotype-directed agents in each oncogenic addiction setting. 
significant impact of the different KRAS mutations subtypes on PFS or OS $[19 \bullet \bullet]$. PD-L1 expression in this population was associated with better outcomes.

More interestingly, some molecular alterations have been shown to have a direct impact, independently on their epidemiologic relationship with tobacco, on the tumor microenvironment. In particularly, STK11 mutations, inactivated in 20 to $30 \%$ of adenocarcinomas, and often associated with KRAS mutations, are associated with non-inflamed tumor microenvironment in a murine model. STK11 inactivation leads to the an increased infiltrate of protumoral neutrophils, a decrease of tumor-infiltrating cells and reduced PD1 and PD-L1 expressions in the tumor microenvironment, both in a KRAS-driven mouse model of lung adenocarcinoma and in cell lines [15]. Furthermore, tumors mutated for both KRAS and TP53 (KP) have a higher mutation load than tumors comutated for KRAS/STK11 (KL), and KP tumors are characterized by a stronger inflammatory response $[20,21]$. In agreement with these preclinical signals, recent data showed that TP53 and STK11 may also accurately stratify patients between responders (57\% ORR for KRAS-P53 tumors) and non-responders (0\% ORR in KRAS-STK11 tumors) in the Checkmate 057 trial [22•].

Regarding EGFR, contradictory results have been reported in predinical studies [23] However, clinical studies have systematically reported disappointing results. Gainor et al. identified a very low response rate $(3.6 \%)$ in patients harboring EGFR mutations treated by immunotherapy and showed an association with non-inflamed tumor microenvironment $[6 \bullet \bullet]$. A recent metaanalysis involving three randomized trials of immunotherapy in pretreated patients (CheckMate 057, Keynote 010, and POPLAR) reported that ICI did not improve overall survival compared with docetaxel in EGFR-mutated patients (HR 1.11;95\% CI 0.80-1.53, $p=0.54$, interaction $p=0.005$ ) [24•]. In a recent phase II study of pembrolizumab in PD-L1 positive, TKI-naïve EGFRmutated patients presented no response even in tumors with high $(\geq 50 \%)$ PDL1 expression highlighting that frontline ICI is inappropriate in EGFR patients [25]. A phase 2, open-label, single-arm trial (ATLANTIC) studied the efficacy of durvalumab, a PD-L1 inhibitor, in pretreated NSCLC including 111 patients with EGFR or ALK alteration [26•]. Patients with EGFR or ALK alteration had previously received standard treatment with tyrosine kinase inhibitors. Amongst the 111 oncogene addicted patients, 77 presented PD-L1 expression in at least $25 \%$ of tumor cells. The aimed response rate was $12.2 \%$ in oncogene addicted patients with PD-L1 expression $>25 \%$ of tumor cells, while in patients with $<25 \%$ PD-L1 expression, the objective response rate was only 3.6\%. Progression-free survival was 1.9 months and was not influenced by PD-L1 expression.

Several explanations to these poor outcomes have been identified, such as an association of EGFR mutation with low TMB and a lack of T cell infiltration $[6 \bullet \bullet, 27]$ while PD-L1 expression is variable $[26 \bullet, 27 \bullet, 28 \bullet]$.

Our IMMUNOTARGET cohort support these findings, with however some differences between sub groups. PFS was 1.4 months for the T790M attached subgroup; 1.8 months for exon 19 deletions; 2.5 for exon 21 mutations, and 2.8 for other mutations $(p<0.0001)$ [21]. In contrast, Negrao et al presented 
appealing data with exon 20 EGFR-mutated tumors compared with classic EGFR-mutations and demonstrated higher OR and disease control rates at 6 and 12 months respectively $13 \%$ and $4 \%$ versus $6 \%$ and $0 \%$ for classic EGFR group) but also higher PFS ( 2.9 vs 1.9 months HR $0.45, p=0.002)$ and OS (HR $0.2, p<0.001)$, compared with common EGFR mutations [29]. Further studies are requested to confirm these differences in outcomes between EGFR mutations subtypes and to adjust these outcomes to tobacco use (more frequent amongst exon 20 mutated patients) and ideally TMB.

\section{ALK, ROS, and other translocations}

Few studies included $A L K$ patients and all reported very poor outcome under ICI. However, the number of patients was usually very low, precluding definitive conclusions $[1,6 \bullet \bullet, 8,26 \bullet]$.

Other translocations, such as RET or ROS1, have been less studied. Poor results were found in the IMMUNOTARGET cohort amongst the concerned population. $A L K, R O S 1$, and RET were analyzed together in a "rearrangement" subgroup, showing a $4.9 \%$ ORR (2/30) [21].

ICI outcomes in RET-rearranged patients have been studied elsewhere. In another retrospective cohort, it was found that RET rearrangements were associated with low TMB and poor response to immunotherapy compared with unselected patients [30•]. Altogether, even if data for ALK /ROS /RET translocations are preliminary and concern a low number of patients, we do not recommend ICI as single agents in patients with $A L K / R O S 1 / R E T$ rearranged NSCLC. Further studies are needed in order to see if these patients will benefit from combination therapies.

The efficacy of immune checkpoint inhibitors in BRAF-mutated NSCLC has also been scarcely studied. Indeed, none of the large clinical trials evaluating antiPD1/anti-PD-L1 agents in advanced NSCLC patients reported results in this specific subgroup. In a retrospective study of 39 BRAF mutant patients, Dudnik et al. showed interesting results of immunotherapy with median PFS of 3.7 and 4.1 months and response rates of 25 and 33\% in patients with V600E and no V600E mutations, respectively. Of note, no significant difference was found between V600E and non-V600E patients in this study [31 $\bullet$.

The IMMUNOTARGET database, however, allowed us to collect data from 35 BRAF-mutated patients who received immunotherapy, of which 17 (48.5\%) were V600E. In this cohort, BRAF mutations were associated with slightly better results compared with EGFR mutations: ORR was $24 \%$ and median PFS of 3.1 months. In BRAF patients, PFS was influenced by smoking, with smokers having better PFS than non-smokers. It therefore seems that immunotherapy should be considered in BRAF-mutated patients, especially if they are smokers. Moreover, non-V600E mutations tended to be associated with better response rates and PFS than V600E mutations, likely due its epidemiologic association

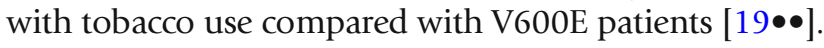
cancers, ORR was 17\% (4/24) and median PFS was 1.9 months (95\% CI 1.7- 
2.7). The authors concluded that responses to ICI may be achieved, but overall clinical efficacy remains modest, suggesting that targeted therapies and chemotherapy must be also favored in this population [32]. The IMMUNOTARGET MET exon 14 cohort proved better outcomes with a median PFS of 4.7 months [20]. This result is supported by another recent limited series $(n=8)$ from Dudnik et al. in which the median PFS was 4.0 months (95\% CI, 2.4-NR). However, the limited number of patients analyzed does not allow us to draw definitive conclusions.

\section{How to obtain better results?}

Except for KRAS patients, response rates to immunotherapy used as a single agent in all oncogene addiction situations were globally shown to be impaired

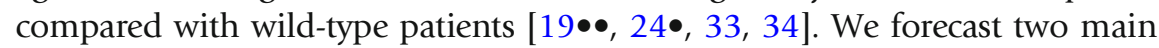
strategies to optimize the integration of immunotherapy in this population: the first one is to identify more reliable biomarkers. The second one is to use them combined with other drugs.

\section{Biomarkers}

\section{PD-L1}

Currently, PD-L1 expression remains the most reliable predictive biomarker to guide PD-1 inhibitors treatment. A recent meta-analysis has clearly demonstrated its interest (OR 2.51), even using a cut-off of 1\% (OR 2.17) [35]. The value of this biomarker has been clearly proved in most of second-line trials but also in the frontline setting, with pembrolizumab showing its superiority over platinum doublet chemotherapy in terms of progression-free but also overall survival in patients whose tumor strongly expressed PD-L1 ( $\geq 50 \%)$, establishing it as a new standard of care [3]. In the IMMUNOTARGET study, PD-L1 expression was significantly associated not only with better outcomes in both EGFR and KRAS subgroups but also in the whole population [22•].

PD-L1 expression is however challenging to interpret in cases of oncogenic addictions. PD-L1 expression may be induced by the oncogenic signaling pathways, but it is not necessarily associated with a strong immune cells infiltration [36, 37] . Furthermore, PD-L1 is a dynamic biomarker, which expression could be induced by radiation therapy, chemotherapies [38, 39], or targeted therapies. Data from archived tissue, often used to guide treatment decisions, are not necessarily representative of the tumor microenvironment at the time of ICI initiation. In conclusion, PD-L1 appears to be less reliable in this context and should be combined with other biomarkers more representative of the tumor microenvironment in order to better select accurate candidates.

\section{Tumor mutation burden}

Beyond PDL1 expression, simple clinical factors have also been explored as predictors of response to immune checkpoint inhibitors. In particular, a lack of tobacco exposure has been associated with lower responsiveness to PD1 blockade $[6 \bullet \bullet, 40]$. One explanation for these findings is that lung cancer in never or 
minimal smokers is generally associated with a low TMB [10], another predictive biomarker of response to immune checkpoint inhibitors, independent of PDL1 expression [41] . Low TMB results in a lack of immunogenic neo-antigens, and thus non-inflamed ("excluded") microenvironment. This is the most likely explanation to the most favorable outcomes observed in the KRAS, BRAF nonV600E, and even MET exon14 patients, alterations that are more frequently observed in smokers. This is consistent with other studies reporting low TMB in EGFR, ALK, ROS1 [42], or RET-driven lung cancers [30•], but higher in KRAS or $B R A F[42]$.

TMB is however, like PD-L1, a dynamic biomarker that may be modified by many anti-cancer treatments. In the KEYNOTE-189 and PACIFIC trials, for example, never-smokers derived benefits from immunotherapy $[2,43]$, likely because chemotherapy and radiation therapy dramatically change the tumor microenvironment, leading to immunogenic cell death with neoantigens release and local and systemic T cells expansions. It is likely that these changes, combined with DNA alterations (and therefore increased TMB), explain the benefit observed in never smokers. TMB analysis can thus be a reliable marker in this population. However, whole-exome sequencing (WES) or the use of broad NGS panels, mandatory for TMB calculation, might be challenging to be translated into routine clinical practice due to the cost, the lack of a standardized panel and cut-off, and the limited availability of tissue [41]. There is thus a need to move beyond $\mathrm{TMB}$ and identify specific genetic determinants of response to PD-1 inhibitors, especially since not all point mutations will result in the genesis of highly immunogenic peptides [44].

The best predictive marker of response in this population combined with TMB may in fact be the tumor-infiltrating lymphocytes (TILs), which defines the notion of "hot" inflammatory tumor and "cold," excluded, tumor.

To increase the probability to obtain sustained disease control in patients with oncogene addiction using ICI, combination therapies may be a promising approach [45]. Activating the patient's immune system during the time of tumor reduction and remission may be the best way to ensure that responses are converted into long-term and durable benefits. Targeted therapy could enhance anti-tumor immune responses by releasing neoantigens and synergistically improve of ICI anti-tumor activity [46]. From a clinical point of view, the combination of targeted agents with immunotherapies is of interest, considering that immunotherapy may transform the important tumor responses achieved with small molecule inhibitors to durable and long-lasting remissions. For example, in melanoma it has been proved that BRAF inhibition could have favorable effects in the tumor microenvironment and it becomes more immunogenic [47]. Several clinical trials investigating combination strategies are currently ongoing, especially for EGFR and ALK. First-line therapy combination of nivolumab and erlotinib showed excellent response rates, even in EGFRTKI pretreated patients, but with a grade 3-4 incidence of adverse-events of $24 \%$ [48]. A phase 1 trial showed an ORR of $77.8 \%$ with gefitinib + durvalumab $(n=10)$ vs $80 \%$ with gefitinib alone followed by the combination of the two drugs $(n=10)$ [49]. Osimertinib and durvalumab also 


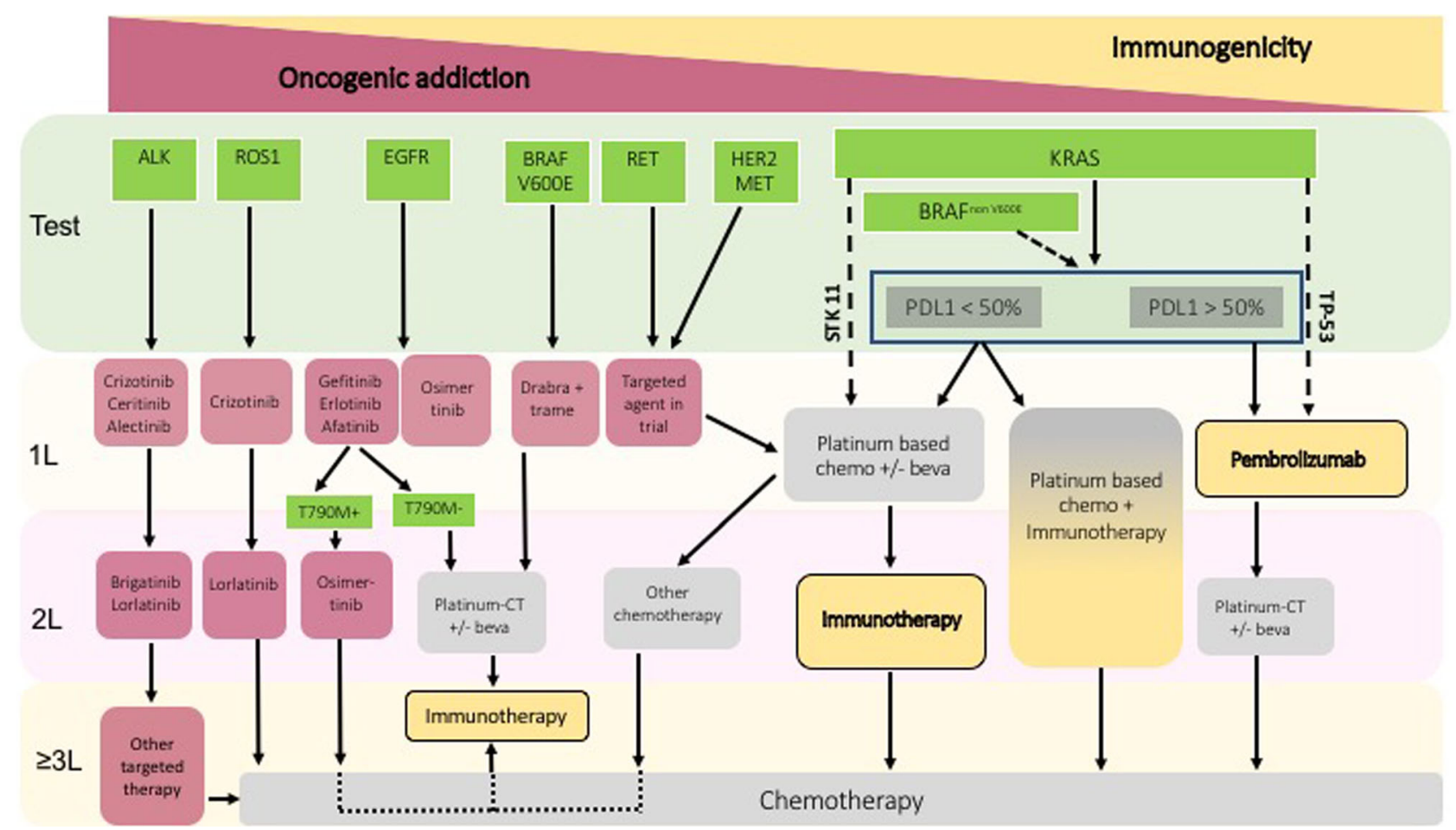

Fig. 3. Proposed algorithm of integration of immunotherapy in the management of patients with oncogenic addiction.

\section{Conclusion}

showed encouraging outcomes in TKI-naïve or resistant EGFR-mutated patients, but this combination seems to strongly potentialize the risk of interstitial pneumonia.

There is also a clear rational for a synergistic effect of ICI, chemotherapy, and anti-VEGF, even in cases of oncogenic addiction. Indeed, cytotoxic chemotherapy exposes the immune system to a high tumor antigen load and the normalization of tumor microvascularisation by anti-VEGF induces an increase in $\mathrm{T}$ lymphocyte infiltration [50]. Such a combination strategy has recently been analyzed in the ImPOWER 150 study (platine-based chemotherapy, bevacizumab, and atezolizumab) and showed significant efficacy in patients with oncogene addictions after failure of all available generations of TKI. The odds ratio for overall survival between the two groups was $0.54(0.29 ; 1.03)$.

Lung cancer is becoming a more diverse disease with regard to management including a wide range of targets and treatment options. In patients with oncogenic addiction, targeted therapy is clearly the most suitable option. The potential interest of immunotherapy to improve patients' outcomes and particularly long-term survival has not been defined yet. Based on published work, we suggest that (i) immunotherapy single agent should be proposed only after exhaustion of much validated treatments (an algorithm according to each driver is proposed in Fig. 3), (ii) a combination of immunotherapy with targeted therapy is of interest provided that we can manage toxicity and find the best sequence, (iii) a combination of immunotherapy with chemotherapy may be 
appealing in patients pretreated with targeted agents. The best way to opt in for the best strategy will depend upon the identification of adequate biomarkers. New basic and clinical research is awaited in this field.

\section{Compliance with Ethical Standards}

Conflict of Interest

The authors declare that they have no conflict of interest.

Human and Animal Rights and Informed Consent

This article does not contain any studies with human or animal subjects performed by any of the authors.

\section{Open Access}

This article is distributed under the terms of the Creative Commons Attribution 4.0 International License (http://creativecommons.org/licenses/by/4.0/), which permits unrestricted use, distribution, and reproduction in any medium, provided you give appropriate credit to the original author(s) and the source, provide a link to the Creative Commons license, and indicate if changes were made.

\section{References and Recommended Reading}

Papers of particular interest, published recently, have been highlighted as:

- Of importance

$\bullet \quad$ Of major importance

1. Herbst RS, Baas P, Kim D-W, Felip E, Pérez-Gracia JL, Han J-Y, et al. Pembrolizumab versus docetaxel for previously treated, PD-L1-positive, advanced nonsmall-cell lung cancer (KEYNOTE-010): a randomised controlled trial. Lancet. 2016;387(10027):1540-50.

2. Antonia SJ, Villegas A, Daniel D, Vicente D, Murakami $\mathrm{S}$, Hui R, et al. Durvalumab after chemoradiotherapy in stage III non-small-cell lung cancer. N Engl J Med. 2017;377(20):1919-29.

3. Reck M, Rodríguez-Abreu D, Robinson AG, Hui $R$, Csőszi T, Fülöp A, et al. Pembrolizumab versus chemotherapy for PD-L1-positive non-small-cell lung cancer. N Engl J Med. 2016;375(19):182333.

4. Weinstein IB. Addiction to oncogenes-the Achilles heal of cancer. Science. 2002;297(5578):63-4.

5. Nagahashi M, Sato S, Yuza K, Shimada Y, Ichikawa H, Watanabe $S$, et al. Common driver mutations and smoking history affect tumor mutation burden in lung adenocarcinoma. J Surg Res. 2018;230:181-5.

6.• Gainor JF, Shaw AT, Sequist LV, Fu X, Azzoli CG Piotrowska Z, et al. EGFR mutations and ALK rearrangements are associated with low response rates to PD-1 pathway blockade in non-small cell lung cancer: a retrospective analysis. Clin Cancer Res. 2016;22(18):4585-9.

Seminal paper reporting the low activity of immunotherapy in NSCLC with oncogenic driver.

7. Barlesi F, Mazieres J, Merlio J-P, Debieuvre D, Mosser J, Lena $\mathrm{H}$, et al. Routine molecular profiling of patients with advanced non-small-cell lung cancer: results of a 1-year nationwide programme of the French Cooperative Thoracic Intergroup (IFCT). Lancet. 2016;387(10026):141526.

8. Borghaei H, Paz-Ares L, Horn L, Spigel DR, Steins M, Ready NE, et al. Nivolumab versus docetaxel in advanced nonsquamous non-small-cell lung cancer. N Engl J Med. 2015;373(17):1627-39.

9. Gainor JF, Rizvi H, Jimenez Aguilar E, Mooradian M, Lydon CA, Anderson D, et al. Response and durability of anti-PD-(L)1 therapy in never- or light-smokers with non-small cell lung cancer (NSCLC) and high PD-L1 expression. J Clin Oncol. 2018;36(15_suppl):9011.

10. Gibbons DL, Byers LA, Kurie JM. Smoking, p53 mutation, and lung cancer. Mol Cancer Res MCR. 2014;12(1):3-13.

11. Jančík S, Drábek J, Radzioch D, Hajdúch M. Clinical relevance of KRAS in human cancers. J Biomed 
Biotechnol [Internet]. 2010 [cited 2019 Feb 7];2010. Available from: https://www.ncbi.nlm.nih.gov/pmc/ articles/PMC2896632/

12. Meng D, Yuan M, Li X, Chen L, Yang J, Zhao X, et al. Prognostic value of K-RAS mutations in patients with non-small cell lung cancer: a systematic review with meta-analysis. Lung Cancer Amst Neth. 2013;81(1):110.

13. Guibert N, Ilie M, Long E, Hofman V, Bouhlel L, Brest $\mathrm{P}$, et al. KRAS Mutations in lung adenocarcinoma: molecular and epidemiological characteristics, methods for detection, and therapeutic strategy perspectives. Curr Mol Med. 2015;15(5):418-32.

14. Tomasini P, Walia P, Labbe C, Jao K, Leighl NB. Targeting the KRAS pathway in non-small cell lung cancer. Oncologist. 2016;21(12):1450-60.

15. Guibert N, Ilie M, Léna H, Didier A, Hofman P, Mazieres J. KRAS and bronchial adenocarcinoma. Between disappointments and hopes. Rev Mal Respir. 2016;33(2):156-64.

16. Miao D, Margolis CA, Vokes NI, Liu D, Taylor-Weiner A, Wankowicz SM, et al. Genomic correlates of response to immune checkpoint blockade in microsatellite-stable solid tumors. Nat Genet. 2018;50(9):1271-81.

17. Rizvi NA, Hellmann MD, Snyder A, Kvistborg P, Makarov V, Havel JJ, et al. Mutational landscape determines sensitivity to PD-1 blockade in non-small cell lung cancer. Science. 2015;348(6230):124-8.

18. Calles A, Liao X, Sholl LM, Rodig SJ, Freeman GJ, Butaney $\mathrm{M}$, et al. Expression of PD-1 and its ligands, PD-L1 and PD-L2, in smokers and never smokers with KRAS-mutant lung cancer. J Thorac Oncol. 2015;10(12):1726-35.

19.• Mazieres J, Drilon AE, Mhanna L, Milia J, Lusque A, Cortot $\mathrm{AB}$, et al. Efficacy of immune-checkpoint inhibitors (ICI) in non-small cell lung cancer (NSCLC) patients harboring activating molecular alterations (ImmunoTarget). J Clin Oncol. 2018;36(15_suppl):901.

The largest cohort reporting efficacy of ICI in patients with oncogenic addiction and presented at the 2018 ASCO annual meeting.

20. Koyama S, Akbay EA, Li YY, Aref AR, Skoulidis F, Herter-Sprie GS, et al. STK11/LKB1 deficiency promotes neutrophil recruitment and proinflammatory cytokine production to suppress T-cell activity in the lung tumor microenvironment. Cancer Res. 2016;76(5):999-1008.

21. Skoulidis F, Byers LA, Diao L, Papadimitrakopoulou VA, Tong P, Izzo J, et al. Co-occurring genomic alterations define major subsets of KRAS-mutant lung adenocarcinoma with distinct biology, immune profiles, and therapeutic vulnerabilities. Cancer Discov. 2015;5(8):86077.

22. Skoulidis F, Goldberg ME, Greenawalt DM, Hellmann MD, Awad MM, Gainor JF, et al. STK11/LKB1 mutations and PD-1 inhibitor resistance in KRAS-mutant lung adenocarcinoma. Cancer Discov. 2018;CD-18-0099.

An important work showing the potential role of STK1/LKB1 in resistance to immunotherapy.
23. Chen N, Fang W, Zhan J, Hong S, Tang Y, Kang S, et al. Upregulation of PD-L1 by EGFR activation mediates the immune escape in EGFR-driven NSCLC: implication for optional immune targeted therapy for NSCLC patients with EGFR mutation. J Thorac Oncol. 2015;10(6):910-23

24. Lee CK, Man J, Lord S, Links M, Gebski V, Mok T, et al. Checkpoint inhibitors in metastatic EGFR-mutated non-small cell lung cancer-a meta-analysis. J Thorac Oncol. 2017;12(2):403-7.

A meta-analysis showing the poor outcome of patients with EGFR mutations and treated in second line with immunotherapy.

25. Lisberg A, Cummings A, Goldman JW, Bornazyan K, Reese N, Wang T, et al. A phase II study of pembrolizumab in EGFR-mutant, PD-L1+, tyrosine kinase inhibitor naïve patients with advanced NSCLC. J Thorac Oncol. 2018;13(8):1138-45.

26. Garassino MC, Cho B-C, Kim J-H, Mazières J, Vansteenkiste J, Lena $\mathrm{H}$, et al. Durvalumab as third-line or later treatment for advanced non-small-cell lung cancer (ATLANTIC): an open-label, single-arm, phase 2 study. Lancet Oncol. 2018;19(4):521-36.

A prospective clinical trial reporting low response rate of EGFRand ALK-positive patients to durvalumab with better results in lung cancer patients with PDL1 $>25 \%$.

27. Dong Z-Y, Zhang J-T, Liu S-Y, Su J, Zhang C, Xie Z, et al. EGFR mutation correlates with uninflamed phenotype and weak immunogenicity, causing impaired response to PD-1 blockade in non-small cell lung cancer. Oncoimmunology. 2017;6(11):e1356145.

28. Zhang M, Li G, Wang Y, Wang Y, Zhao S, Haihong P, et al. PD-L1 expression in lung cancer and its correlation with driver mutations: a meta-analysis. Sci Rep. 2017 [cited 2018 Jul 10];7(1).

29. Negrao MV, Reuben A, Robichaux JP, Le X, Nilsson MB, $\mathrm{Wu} \mathrm{C}$, et al. Association of EGFR and HER-2 exon 20 mutations with distinct patterns of response to immune checkpoint blockade in non-small cell lung cancer. J Clin Oncol. 2018;36(15_suppl):9052.

30. Sabari JK, Offin MD, Wu SL, Ni A, Halpenny D, Montecalvo J, et al. RET-rearranged lung cancers: immunophenotype and response to immunotherapy. J Clin Oncol. 2018;36(15_suppl):9034.

A study focusing on RET-positive patients treated with immunotherapy.

31. Dudnik E, Peled N, Nechushtan H, Wollner M, Onn A, Agbarya A, et al. BRAF mutant lung cancer: programmed death ligand 1 expression, tumor mutational burden, microsatellite instability status, and response to immune check-point inhibitors. J Thorac Oncol. 2018;13(8):1128-37.

A study focusing on BRAF-positive patients treated with immunotherapy.

32. Sabari JK, Montecalvo J, Chen R, Dienstag JA, Mrad C, Bergagnini I, et al. PD-L1 expression and response to immunotherapy in patients with MET exon 14-altered non-small cell lung cancers (NSCLC). J Clin Oncol. 2017;35(15_suppl):8512. 
33. Remon J, Hendriks LE, Cabrera C, Reguart N, Besse B. Immunotherapy for oncogenic-driven advanced nonsmall cell lung cancers: is the time ripe for a change? Cancer Treat Rev. 2018;71:47-58.

34. Gettinger S, Politi K. PD-1 Axis inhibitors in EGFR- and ALK-driven lung cancer: lost cause? Clin Cancer Res Off J Am Assoc Cancer Res. 2016;22(18):4539-41.

35. Khunger M, Hernandez AV, Pasupuleti V, Rakshit $S$, Pennell NA, Stevenson J, et al. Programmed cell death 1 (PD-1) ligand (PD-L1) expression in solid tumors as a predictive biomarker of benefit from PD-1/PD-L1 axis inhibitors: a systematic review and meta-analysis. JCO Precis Oncol. 2017;1(1):1-15.

36. D'Incecco A, Andreozzi M, Ludovini V, Rossi E, Capodanno A, Landi L, et al. PD-1 and PD-L1 expression in molecularly selected non-small-cell lung cancer patients. Br J Cancer. 2015;112(1):95-102.

37. Ota K, Azuma K, Kawahara A, Hattori S, Iwama E, Tanizaki J, et al. Induction of PD-L1 expression by the EML4-ALK oncoprotein and downstream signaling pathways in non-small cell lung cancer. Clin Cancer Res. 2015;21(17):4014-21.

38. Han JJ, Kim D-W, Koh J, Keam B, Kim TM, Jeon YK, et al. Change in PD-L1 expression after acquiring resistance to gefitinib in EGFR-mutant non-small-cell lung cancer. Clin Lung Cancer. 2016;17(4):263270.e2.

39. Gong X, Li X, Jiang T, Xie H, Zhu Z, Zhou F, et al. Combined radiotherapy and anti-PD-L1 antibody synergistically enhances antitumor effect in non-small cell lung cancer. J Thorac Oncol. 2017;12(7):1085-97.

40. Kim JH, Kim HS, Kim BJ. Prognostic value of smoking status in non-small-cell lung cancer patients treated with immune checkpoint inhibitors: a meta-analysis. Oncotarget. 2017;8(54):93149-55.

41. Hellmann MD, Ciuleanu T-E, Pluzanski A, Lee JS, Otterson GA, Audigier-Valette C, et al. Nivolumab plus ipilimumab in lung cancer with a high tumor mutational burden. N Engl J Med. 2018;378(22):2093-104.

42. Spigel DR, Schrock AB, Fabrizio D, Frampton GM, Sun J, He J, et al. Total mutation burden (TMB) in lung cancer (LC) and relationship with response to PD-1/PD-L1 targeted therapies. J Clin Oncol. 2016;34(15_suppl):9017. 43. Gandhi L, Rodríguez-Abreu D, Gadgeel S, Esteban E, Felip E, De Angelis F, et al. Pembrolizumab plus chemotherapy in metastatic non-small-cell lung cancer. N Engl J Med. 2018;378(22):2078-92.
44. Chen DS, Mellman I. Elements of cancer immunity and the cancer-immune set point. Nature. 2017;541(7637):321-30.

45. Karachaliou N, Gonzalez-Cao M, Sosa A, Berenguer J, Bracht JWP, Ito $M$, et al. The combination of checkpoint immunotherapy and targeted therapy in cancer. Ann Transl Med. 2017;5(19):388.

46. Pilotto S, Molina-Vila MA, Karachaliou N, Carbognin L, Viteri S, González-Cao M, et al. Integrating the molecular background of targeted therapy and immunotherapy in lung cancer: a way to explore the impact of mutational landscape on tumor immunogenicity.

Transl Lung Cancer Res. 2015;4(6):721-7.

47. Wilmott JS, Long GV, Howle JR, Haydu LE, Sharma $\mathrm{RN}$, Thompson JF, et al. Selective BRAF inhibitors induce marked T-cell infiltration into human metastatic melanoma. Clin Cancer Res. 2012;18(5):1386-94.

48. Rizvi NA, Chow LQM, Borghaei H, Shen Y, Harbison C, Alaparthy S, et al. Safety and response with nivolumab (anti-PD-1; BMS-936558, ONO-4538) plus erlotinib in patients (pts) with epidermal growth factor receptor mutant (EGFR MT) advanced NSCLC. J Clin Oncol. 2014;32(15_suppl):8022.

49. Gibbons DL, Chow LQ, Kim D-W, Kim S-W, Yeh T, Song X, et al. $57 \mathrm{O}$ efficacy, safety and tolerability of MEDI4736 (durvalumab [D]), a human IgG1 antiprogrammed cell death-ligand-1 (PD-L1) antibody, combined with gefitinib (G): a phase I expansion in TKI-naïve patients (pts) with EGFR mutant NSCLC. J Thorac Oncol. 2016;11(4 Suppl):S79.

50. Hegde PS, Wallin JJ, Mancao C. Predictive markers of anti-VEGF and emerging role of angiogenesis inhibitors as immunotherapeutics. Semin Cancer Biol. 2018;52(Pt 2):117-24.

51. Gainor JF, Shaw AT. Emerging paradigms in the development of resistance to tyrosine kinase inhibitors in lung cancer. J Clin Oncol. 2013;31(31):3987-96.

\section{Publisher's Note}

Springer Nature remains neutral with regard to jurisdictional claims in published maps and institutional affiliations. 